\title{
Inpainting Ideas for Image Compression
}

\author{
Joachim Weickert \\ Faculty of Mathematics and Computer Science \\ Saarland University, Building E1.1 \\ 66041 Saarbruecken, Germany \\ weickert@mia.uni-saarland.de \\ http://www.mia.uni-saarland.de/weickert
}

\begin{abstract}
In the last decade, partial differential equations (PDEs) have demonstrated their usefulness for so-called inpainting problems, where missing image information is recovered by interpolating data from the neighbourhood. For inpainting problems, however, usually a large fraction of the image data is available.

We extend these ideas to a much more challenging task, namely PDEbased lossy image compression. To this end, only a very small amount of data are kept, and the remaining data are restored using PDE-based interpolation. This gives rise to three interdependent questions: (1) What are the most useful PDEs for this task? (2) Which are the best points for being kept? (3) How can these data be encoded in an efficient way?

In this talk, recent results from our group will be presented. For the linear diffusion operator, a method for optimal point selection is described. In the anisotropic diffusion setting, a tree-based subdivision method is used to encode relevant pixels in a compact way. Our experiments demonstrate that PDE-based image compression may give better results than JPEG and even JPEG2000, in particular if images are not dominated by texture and high compression rates are required.
\end{abstract}

